\title{
Review of: "SNP and Haplotype Regional Heritability Mapping (SNHap-RHM): joint mapping of common and rare variation affecting complex traits"
}

\author{
Olivier Gervais \\ 1 Nihon University
}

Potential competing interests: The author(s) declared that no potential competing interests exist.

This manuscript explores a novel analytical approach (SNHap-RHM), which estimates the heritability of a region of the genome by capturing both SNP-based effects and haplotype-based effects. The authors compare the results of the SNHap-RHM approach with already established methods (SNP-RHM and HapRHM) by using simulation data as well as real-world data.

Overall, the analyses were reasonable and the results clearly presented, but some additional details would have been valuable at several points.

1. From your analysis of Height and MDD (Figures 5 and 6), the SNP-RHM and Hap-RHM methods appear to have higher statistical power (lower p-values) for the detection of susceptibility loci than the SNHapRHM approach (For Height: ADAMTSL3, GRM4, NCAPG, HHIP; for MDD: DCC, MYRIP). For instance, several of the genes that cleared the genome-wide significance threshold with SNP-RHM are only detected at the suggestive level with SNHap-RHM (ADAMTSL3, GRM4). This point, as well as the reasons for this result, should be addressed in more detail in the discussion.

2. L349-354: Related to the point above, you state that "The results for height show that nine regions passed the Bonferroni-corrected genome-wide significance threshold in the analysis using SNP-RHM. No region was genome-wide significant for height when analysed with Hap-RHM. Furthermore, these associations still come up when SNPs and haplotypes in those regions are analysed jointly using SNHapRHM." While these statements are technically true, I find the paragraph misleading as it seems to imply that SNHap-RHM is as effective as the other methods implemented separately, which does not seem to be the case given the results presented (see my comment above); in particular, "still come up" is very general and vague. The paragraph should be rephrased.

3. Some discussion about the computation time of the SNHap-RHM approach (with 2 regional GRMs) vs. SNP-RHM/Hap-RHM would be useful.

4. L340-341: "The heritability estimates for height and MDD, calculated using the whole-genome GRM, were $81.4 \%$ and $13.8 \%$ respectively". The SE estimates should be added.

5. L379: "A SNP in this gene is reported to be associated with brain processing speed". Add the SNP rsID.

6. L382: "Also, a SNP in the MYRIP gene region is associated with sleep duration". Add the SNP rsID. 
